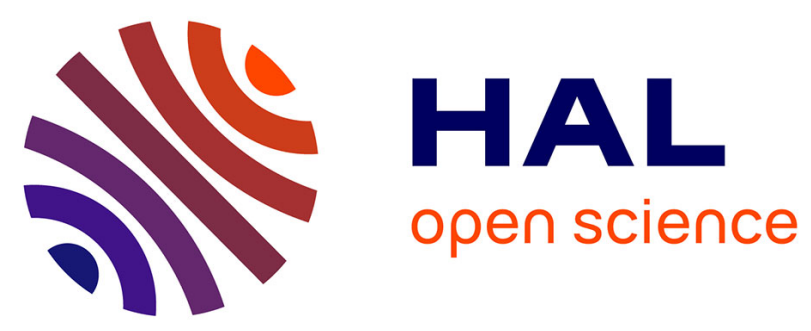

\title{
Inband Full-Duplex D2D Communications Underlaying Uplink Networks with Mutual SIC NOMA
}

\author{
Antoine Kilzi, Joumana Farah, Charbel Abdel Nour, Catherine Douillard
}

\section{To cite this version:}

Antoine Kilzi, Joumana Farah, Charbel Abdel Nour, Catherine Douillard. Inband Full-Duplex D2D Communications Underlaying Uplink Networks with Mutual SIC NOMA. PIMRC 2020: IEEE 31st Annual International Symposium on Personal, Indoor and Mobile Radio Communications, IEEE, Aug 2020, London, United Kingdom. pp.1-7, 10.1109/PIMRC48278.2020.9217153 . hal-03563253

\section{HAL Id: hal-03563253 \\ https://imt-atlantique.hal.science/hal-03563253}

Submitted on 9 Feb 2022

HAL is a multi-disciplinary open access archive for the deposit and dissemination of scientific research documents, whether they are published or not. The documents may come from teaching and research institutions in France or abroad, or from public or private research centers.
L'archive ouverte pluridisciplinaire $\mathbf{H A L}$, est destinée au dépôt et à la diffusion de documents scientifiques de niveau recherche, publiés ou non, émanant des établissements d'enseignement et de recherche français ou étrangers, des laboratoires publics ou privés. 


\title{
Inband Full-Duplex D2D Communications Underlaying Uplink Networks with Mutual SIC NOMA
}

\author{
Antoine $\operatorname{Kilzi}^{(1)}$, Joumana $\operatorname{Farah}^{(2)}$, Charbel Abdel Nour ${ }^{(1)}$, Catherine Douillard ${ }^{(1)}$ \\ (1) IMT Atlantique, Department of Electronics, Lab-STICC - UMR 6285 \\ Technopôle Brest Iroise, CS 83818 - 29238 Brest Cedex, France \\ ${ }^{(2)}$ Department of Electricity and Electronics, Faculty of Engineering, \\ Lebanese University, Roumieh, Lebanon
}

\begin{abstract}
This paper studies the combination of full-duplex (FD) and half-duplex (HD) device-to-device (D2D) communications while underlaying a cellular system. Non-orthogonal multiple access (NOMA) is used to manage the interference between devices and cellular users using mutual successive interference cancellation (SIC) and to boost the performance of D2D underlay systems, which are mainly interference-limited. For this purpose, we derive the conditions allowing for the application of mutual SIC in FD-D2D and HD-D2D systems. We compare the performance of the proposed strategy against state-of-theart, where SIC is not applied between D2D devices and cellular users. The results show that important gains can be achieved by using NOMA in this context and highlight the importance of self-interference (SI) cancellation factors for determining the best transmission mode.
\end{abstract}

Index Terms-Non-orthogonal multiple access, D2D, mutual SIC, full duplex, half duplex, residual self interference.

\section{INTRODUCTION}

The growth of the number of connected devices has hit unprecedented highs in the last few years, and this trend is set to last for the years to come [1]. Indeed, their number is expected to be more than three times the world population by 2023. The corresponding network densification, coupled with the expected increase in data traffic and the limited available spectrum, will require novel efficient solutions to supply the ever increasing demand. Full-duplex (FD) communication combined with device-to-device (D2D) communication represent an attractive solution to leverage the challenges of future generation networks.

D2D enables direct communication between nearby devices with little to no information transiting through the network base stations (BS) [2]. The D2D communication rate, in this regard, is alleviated from the network which can use the freed capacity to serve other users, hence increasing the number of accommodated devices. FD communication enables a node to send and receive simultaneously using the same frequency resource. In theory, the achieved gain is a two-fold increase in spectral efficiency (SE) compared to half-duplex (HD) send-then-receive systems. However, a self interference (SI) is incurred due to the transmitted signal looping back into the receiver, thus limiting its appeal compared to HD to the point where this latter may even outperform FD in some cases.
Nonetheless, the improvement in antenna architecture and in SI cancellation circuitry dramatically reduces the residual self interference (RSI) [3], [4], pushing FD to be adopted in 5G.

The increasing demand for connected devices pushed research into the direction of non-orthogonal multiple access (NOMA) techniques. In NOMA, multiple devices can share the same time and frequency resource but are differentiated in a non-orthogonal dimension, e.g. code-domain and powerdomain NOMA (PD-NOMA) [5]. In the latter, superposition coding of the user signals is used at the transmitter, and successive interference cancellation (SIC) is performed at the receiver side. The message with the highest power is decoded first and then subtracted from the total received signal, then the second highest power message is extracted and so on until the user decodes its own message [6-9].

In [10], a NOMA FD-D2D system is proposed, where FD is used at the level of the strong NOMA user in D2D relay mode to help and assist the weak NOMA user. This FD-D2D aided cooperative NOMA scheme can perform better than regular NOMA, depending on the RSI and channel constraints. This led to the elaboration of an adaptive multiple access scheme efficiently switching back to conventional NOMA when it is favorable. In [11], D2D is studied in the context of vehicle-to-vehicle (V2V) communication where strict latency conditions must be satisfied. The formulated problem accounts for the latency constraints and the proposed heuristic solution for the resource block allocation relies only on slow fading channel information. The same approach is followed in [12] to transform the latency and reliability requirements of vehicleto-everything (V2X) communications into optimization constraints. Moreover, non-orthogonal access for vehicle-type users is allowed and the optimization problem is formulated to maximize the cellular user $(\mathrm{CU})$ rates while satisfying the latency and reliability constraints of V2X users.

The study in [13] considers resource block assignment and power allocation (PA) for the combination of NOMA with D2D communications in underlay mode. HD is used in the D2D pairs, and CUs are grouped in downlink NOMA clusters. However, NOMA SIC is not used to decode the interfering signals of the colocated D2D pairs. The same is true for [14], where additional power constraints are introduced to D2D 
pairs for the sake of the CUs SIC decoding order conditions. The work in [15] introduces the concept of D2D group, where a D2D transmitter communicates with multiple D2D receivers via NOMA. Sub-channel allocation is conducted using manyto-one matching for CU-D2D grouping, and optimal PA is approximated iteratively via successive convex approximation. In [16], joint D2D-CU grouping and PA is conducted by successively applying the Kuhn-Munkres technique for channel allocation, while optimal PA is obtained using the KarushKuhn-Tucker conditions. The objective of these works is to maximize energy efficiency and/or system throughput under $\mathrm{CU}$ target rate constraints. In all the preceding studies, NOMA is applied either between the CU users [13], [14], or between users of the same D2D group [15], [16]. In our previous works [17-[19], we introduced the concept of mutual SIC, where we showed that the signals of two or more users multiplexed in NOMA, and powered by distributed antennas, can be decoded and removed at the level of every user in the NOMA cluster.

To the best of our knowledge, NOMA has not been applied between CU and D2D users; and even less for the case of FDD2D underlay. We hear by NOMA between D2D and CU, the intervention of the $\mathrm{BS}$ in signaling and power control to enable the interference cancellation of D2D signals at the level of the $\mathrm{BS}$, and the cancellation of the CU message at the level of the D2D pair. This is done by applying mutual SIC between the D2D pair and the BS. The necessary SIC constraints in terms of power multiplexing conditions (PMC) and rate conditions are derived. Also, the PA problems are formulated and solved for the cases of FD and HD transmissions, as well as for SIC-enabled and disabled scenarios. The paper is organized as follows: section II presents the considered system model and formulates the PA problem for FD and HD with and without mutual SIC. In section III], the conditions of mutual SIC for FD-D2D are derived, and in section IV constraint reduction of the mutual SIC PA problem is performed. In section $\mathrm{V}$, mutual SIC PA is solved for the case of HD transmission. Simulation results are presented in section $\mathrm{VI}$, and conclusions are drawn in section VII.

\section{SySTEM MODEL}

Consider a cellular network consisting of one D2D pair transmitting over an uplink (UL) channel previously allocated to a CU $u$. The D2D pair can transmit either in HD or in FD mode, while the CU is always in HD. A schematic of the network is presented in Fig. 1, where $d_{1}$ and $d_{2}$ are the D2D users transmitting in FD mode. The interference channel gains between the $\mathrm{CU}$, on the one hand, and $d_{1}$ and $d_{2}$ on the other hand are denoted by $h_{d_{1}, u}$ and $h_{d_{2}, u}$ respectively. The direct link between the CU $u$ and the BS $b$ has a gain denoted by $h_{b, u}$. The signal $s_{u}$, transmitted by $u$ with power $P_{u}$, reaches the BS with a power level $P_{u} h_{b, u}$, and causes an interference level of $P_{u} h_{d_{1}, u}$ and $P_{u} h_{d_{2}, u}$ at $d_{1}$ and $d_{2}$ respectively. Each device $d_{i}$ of the D2D pair can transmit a signal $s_{i}$ of power $P_{i}$ to the other D2D user and suffers from both the interference of user $u$ and its RSI power $\eta_{i} P_{i}$, with $\eta_{i}$ the SI cancellation capability. The D2D channel gain is denoted by $h_{d}$ and the interference channel gains from $d_{1}$ and $d_{2}$ to the BS are denoted by $h_{b, d_{1}}$ and $h_{b, d_{2}}$. In this work, we assume that the $\mathrm{BS}$ has perfect knowledge of the long-term evolution of the different channel gains, through signaling exchange between the different entities. The BS performs resource allocation based on these estimated channel gains and instructs the $\mathrm{CU}$ and D2D pair of the required transmit power according to the selected transmission scenario.

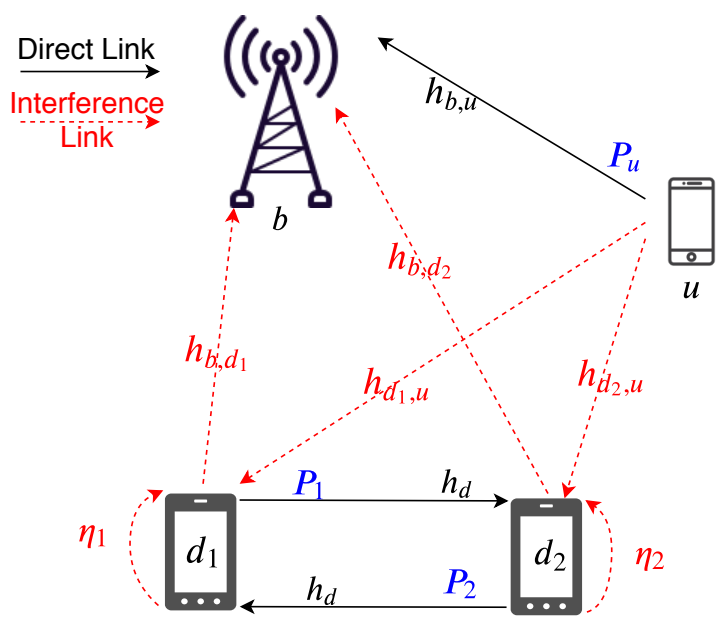

Figure 1: FD inband underlay communication sharing the UL resource of a CU.

\section{A. PA problem formulation for FD and HD scenarios without mutual interference cancellation}

The idea behind inband underlay D2D lies in reusing licensed spectrum to allow for the communication of more users without affecting the quality of service (QoS) of the CU. Therefore, the objective of all the presented transmission scenarios is to maximize the D2D rate while maintaining the QoS requirement of user $u$. The generic problem can be written as follows:

$$
\left\{P_{1}^{*}, P_{2}^{*}, P_{u}^{*}\right\}=\arg \max R_{D 2 D},
$$

$$
\begin{array}{lll}
\text { s.t. } & R_{u} \geq R_{u, \text { min }}, \quad \text { (1a) } & P_{u} \leq P_{u, \text { max }}, \\
& P_{1} \leq P_{1, \text { max }}, \quad \text { (1b) } & P_{2} \leq P_{2, \text { max }},
\end{array}
$$

where $P_{u, \max }, P_{1, \max }$, and $P_{2, \max }$ are the maximum transmit powers of users $u, d_{1}$ and $d_{2}$ respectively, $R_{u, \text { min }}$ is the minimum target rate of user $u$ with $R_{u}$ its achieved rate, and $R_{D 2 D}$ is the D2D rate, sum of the achieved rates by $d_{1}$ $\left(R_{d_{1}}\right)$ and $d_{2}\left(R_{d_{2}}\right)$. The problem formulation in (1) is used throughout the paper as a generic form for all transmission scenarios with the proper modifications in the rate expressions.

1) FD-NoSIC: In FD, $d_{1}$ and $d_{2}$ transmit simultaneously, thus they both suffer from RSI. The signal-to-interference- 
plus-noise ratios (SINRs) at the level of the BS and the D2D users are given by:

$$
\begin{aligned}
& \operatorname{SINR}_{b}=\frac{P_{u} h_{b, u}}{P_{1} h_{b, d_{1}}+P_{2} h_{b, d_{2}}+\sigma^{2}}, \\
& \operatorname{SINR}_{d_{1}}=\frac{P_{2} h_{d}}{P_{u} h_{d_{1}, u}+\eta_{1} P_{1}+\sigma^{2}}, \\
& \operatorname{SINR}_{d_{2}}=\frac{P_{1} h_{d}}{P_{u} h_{d_{2}, u}+\eta_{2} P_{2}+\sigma^{2}},
\end{aligned}
$$

with $\sigma^{2}$ the additive Gaussian noise power. The achieved rates are expressed according to the Shannon capacity theorem:

$$
\begin{gathered}
R_{u}=B \log _{2}\left(1+S I N R_{b}\right), \\
R_{d_{1}}=B \log _{2}\left(1+S I N R_{d_{1}}\right), \quad R_{d_{2}}=B \log _{2}\left(1+S I N R_{d_{2}}\right),
\end{gathered}
$$

with $B$ the bandwidth of the UL resource initially allocated to $u$. Due to the interference terms in (2), (1) is a non-convex problem. To solve it, a geometrical representation can be used, leading to the analytical global solution in [20]. This method is adopted in our work to derive the results of the FD-NoSIC scenario in the performance assessment section.

2) HD-NoSIC: The time slot is now divided into two equal half time slots where $d_{1}$ and $d_{2}$ alternately transmit and receive information. To maximize the total D2D rate, the optimization is conducted in the two half time slots. In the first half, $d_{1}$ transmits information $\left(P_{2}=0\right)$. In $(1)$, the objective function and $\mathrm{CU}$ rate are now:

$$
\begin{gathered}
R_{D 2 D, 1}=R_{d_{2}}=B \log _{2}\left(1+\frac{P_{1} h_{d}}{P_{u, 1} h_{d_{2}, u}+\sigma^{2}}\right), \\
R_{u, 1}=B \log _{2}\left(1+\frac{P_{u, 1} h_{b, u}}{P_{1} h_{b, d_{1}}+\sigma^{2}}\right) .
\end{gathered}
$$

In the first half time slot, problem (1) is constrained only by eqs. (1a) to (1c). Note that $P_{u, 1}$ is the transmit power of $u$ during the first half time slot. $R_{D 2 D, 1}$ is strictly increasing with $P_{1}$ and decreasing with $P_{u, 1}$; therefore, to maximize $R_{D 2 D, 1}=R_{d_{2}}, P_{1}$ should be increased and $P_{u, 1}$ decreased as long as $R_{u, 1}$ satisfies the minimum rate condition. Consequently, $P_{1}$ should be increased as much as possible and then $P_{u, 1}$ is obtained as a function of $P_{1}\left(P_{u, 1}=f\left(P_{1}\right)\right)$ by enforcing an equality between $R_{u, 1}$ and $R_{u, \min }$. If for $P_{1}=$ $P_{1, \text { max }}, f\left(P_{1, \text { max }}\right) \leq P_{u, \text { max }}$, the couple $\left(P_{1, \text { max }}, f\left(P_{1, \text { max }}\right)\right)$ is retained as the $\left(P_{1}, P_{u, 1}\right)$ solution; otherwise, the couple $\left(f^{-1}\left(P_{u, \max }\right), P_{u, \max }\right)$ delivers the best solution. The same reasoning is applied for the second half time slot (where $P_{1}=0$ ) to maximize $R_{D 2 D, 2}=R_{d_{1}}$. The total D2D and user $u$ rates are given by:

$$
\begin{gathered}
R_{u}=\frac{1}{2} R_{u, 1}+\frac{1}{2} R_{u, 2}, \\
R_{D 2 D}=\frac{1}{2} R_{d_{1}}+\frac{1}{2} R_{d_{2}} .
\end{gathered}
$$

B. PA problem formulation for $H D$ and FD with mutual SIC (HD-SIC and FD-SIC)

Using a SIC receiver at the level of the BS and the D2D users, interfering signals can be decoded then subtracted from the received signal, canceling thereby the interference in both FD and HD scenarios. In the case of FD, the BS can decode and subtract successively $s_{1}$ then $s_{2}$, or reversely, before proceeding to the decoding of $s_{u}$; hence, two decoding orders are possible. Users $d_{1}$ and $d_{2}$ can also remove the interference of $u$, leading to the following SINR expressions:

$S I N R_{d_{1}}=\frac{P_{2} h_{d}}{\eta_{1} P_{1}+\sigma^{2}}, S I N R_{d_{2}}=\frac{P_{1} h_{d}}{\eta_{2} P_{2}+\sigma^{2}}, S I N R_{b}=\frac{P_{u} h_{b, u}}{\sigma^{2}}$.

The SINRs are replaced in eqs. (3) and (4) to obtain $R_{u}$ and $R_{D 2 D}=R_{d_{1}}+R_{d_{2}}$. For the case of HD, the SINRs in the first half time slot are:

$$
\operatorname{SINR}_{d_{2}}=P_{1} h_{d} / \sigma^{2}, \quad \quad \operatorname{SINR}_{b}=P_{u} h_{b, u} / \sigma^{2} .
$$

In the second half time slot, $S I N R_{b}$ is the same and $S I N R_{d_{1}}=$ $P_{2} h_{d} / \sigma^{2}$. Problem (1) is now reformulated in each time slot by expressing the rates using the present SINRs. However, additional constraints relative to the SIC feasibility must be added to the problem. They are derived next.

\section{DERIVATION OF THE SIC CONDITIONS FOR FD MUTUAL SIC}

In this scenario, we are looking for the conditions that allow $d_{1}$ to decode $s_{u}, d_{2}$ to decode $s_{u}$, and $b$ to decode $s_{1}$ and $s_{2}$. As already mentioned, two decoding orders are possible at the level of $b$.

\section{A. First decoding order: $b$ decodes $s_{2}$ then $s_{1}$}

We first start by studying the mutual SIC constraints between $b$ and $d_{1}$ (as a receiver). Let $\operatorname{SINR}_{i}^{s_{j}}$ be the SINR of the signal $s_{j}$ at the level of user $i$ ( $i$ is either $d_{1}, d_{2}$ or $b$, and $j$ is either 1,2 or $u$ ). For $b$ to successfully decode the signal $s_{2}$ transmitted by $d_{2}$ to $d_{1}$, the rate of $s_{2}$ at the level of $b$ must be greater than the rate of $s_{2}$ at the level of $d_{1}$. Thus, we must have:

$$
\begin{gathered}
\operatorname{SINR}_{b}^{S_{2}}>\operatorname{SINR}_{d_{1}}^{s_{2}}, \\
\frac{P_{2} h_{b, d_{2}}}{\sigma^{2}+P_{1} h_{b, d_{1}}+P_{u} h_{b, u}}>\frac{P_{2} h_{d}}{\sigma^{2}+P_{1} \eta_{1}+P_{u} h_{d_{1}, u}}
\end{gathered}
$$

By neglecting the noise power compared to the interfering terms, the SIC condition is given by:

$$
P_{1}\left(h_{b, d_{2}} \eta_{1}-h_{d} h_{b, d_{1}}\right)+P_{u}\left(h_{d_{1}, u} h_{b, d_{2}}-h_{d} h_{b, u}\right)>0 .
$$

In addition to condition (7), the PMCs must be verified. The PMC ensures that the signal to be decoded first at the level of a receiver must have a higher power level than the remaining signals combined [19]. Since $b$ decodes $s_{2}$ first, then we have the following PMC for the decoding of $s_{2}$.

$$
P_{2} h_{b, d_{2}}>P_{1} h_{b, d_{1}}+P_{u} h_{b, u}
$$

For $d_{1}$ to remove the interference of $s_{u}$ prior to retrieving $s_{2}$, we must have $S I N R_{d_{1}}^{s_{u}}>S I N R_{b}^{S_{u}}$, which leads to:

$$
P_{1}\left(h_{d_{1}, u} h_{b, d_{1}}-h_{b, u} \eta_{1}\right)+P_{2}\left(h_{d_{1}, u} h_{b, d_{2}}-h_{b, u} h_{d}\right)>0,
$$

and the corresponding PMC is:

$$
P_{u} h_{d_{1}, u}>P_{2} h_{d}+P_{1} \eta_{1}
$$


Regarding the mutual SIC between the receivers $b$ and $d_{2}$, the decoding of $s_{1}$ at the level of $b$ requires $S I N R_{b}^{S_{1}}$ to be greater than $\operatorname{SINR}_{d_{2}}^{S_{1}}$ :

$$
\begin{gathered}
\frac{P_{1} h_{b, d_{1}}}{\sigma^{2}+P_{u} h_{b, u}}>\frac{P_{1} h_{d_{2}, d_{1}}}{\sigma^{2}+P_{2} \eta_{2}+P_{u} h_{d_{2}, u}}, \\
P_{2} h_{b, d_{1}} \eta_{2}>P_{u}\left(h_{b, u} h_{d}-h_{d_{2}, u} h_{b, d_{1}}\right) .
\end{gathered}
$$

Note that $S I N R_{b}^{S_{1}}$ does not include $P_{2}$ since $s_{2}$ is decoded and canceled prior to $s_{1}$. The corresponding PMC is given by:

$$
P_{1} h_{b, d_{1}}>P_{u} h_{b, u} .
$$

At the level of $d_{2}, S I N R_{d_{2}}^{S_{u}}$ must be greater than $\operatorname{SINR}_{b}^{S_{u}}$ to decode and subtract $s_{u}$ before retrieving $s_{1}$. This yields the following condition:

$$
P_{1}\left(h_{b, d_{1}} h_{d_{2}, u}-h_{d} h_{b, u}\right)>P_{2} \eta_{2} h_{b, u}
$$

Finally, the PMC at the level of $d_{2}$ is given by:

$$
P_{u} h_{d_{2}, u}>P_{1} h_{d}+P_{2} \eta_{2}
$$

\section{B. Second decoding order: $b$ decodes $s_{1}$ then $s_{2}$}

Following the same reasoning as in Section III-A for the case where $s_{1}$ is decoded before $s_{2}$ at the level of $b$, the PMC and rate constraints for a full SIC between $d_{1}$ and $b$, and $d_{2}$ and $b$, are obtained and listed below:

$$
\begin{gathered}
P_{1} \eta_{1} h_{b, d_{2}}>P_{u}\left(h_{b, u} h_{d}-h_{b, d_{2}} h_{b, d_{1}}\right) \\
P_{2}\left(h_{d_{1}, u} h_{b, d_{2}}-h_{u, b} h_{d}\right)>h_{u, b} \eta_{1} P_{1} \\
P_{2}\left(h_{b, d_{1}} \eta_{2}-h_{b, d_{2}} h_{d}\right)+P_{u}\left(h_{b, d_{2}} h_{b, d_{1}}-h_{b, u} h_{d}\right)>0 \\
P_{1}\left(h_{d_{2}, u} h_{b, d_{1}}-h_{d} h_{u, b}\right)+P_{2}\left(h_{d_{2} b} h_{d_{2}, u}-h_{u, b} \eta_{2}\right)>0 \\
P_{2} h_{b, d_{2}}>P_{u} h_{b, u} \\
P_{u} h_{d_{1}, u}>P_{2} h_{d}+P_{1} \eta_{1} \\
P_{1} h_{b, d_{1}}>P_{u} h_{b, u}+P_{2} h_{b, d_{2}} \\
P_{u} h_{d_{2}, u}>P_{1} h_{d}+P_{2} \eta_{2}
\end{gathered}
$$

Problem (1) now includes, in addition to constraints eqs. (1a) to $\sqrt{1 d}$, eight new constraints that express the full SIC feasibility (either equations (7) to (14) or (15) to (22), depending on the decoding order). In the next section, we analyze the interplay between SIC rate conditions and PMCs in order to remove redundant constraints from problem (1).

\section{PRoblem Simplification OF FD-SIC BY CONSTRAINT REDUCTION}

Consider the first decoding order at the level of $b$ where $s_{2}$ is decoded before $s_{1}$. The PMCs for the decoding of $s_{1}$ at the level of $b$ and of $s_{u}$ at the level of $d_{2}$ are given by (12) and (14). By multiplying (12) by $h_{d_{2}, u}$ and adding it to (14) multiplied by $h_{b, u}$, one can eliminate $P_{u}$ to obtain:

$$
P_{1}\left(h_{b, d_{1}} h_{d_{2}, u}-h_{d} h_{b, u}\right)>P_{2} \eta_{2} h_{b, u},
$$

which is the SIC condition (13) to remove $s_{u}$ at the level of $d_{2}$. Also, eliminating $P_{1}$ from the two PMCs by means of adding (12) multiplied by $h_{d}$ to (14) multiplied by $h_{b, d_{1}}$ yields (11). Consequently, the PMCs for the decoding of $s_{1}$ at the level of $b$, and $s_{u}$ at the level of $d_{2}$ imply their counterpart rate conditions. Moreover, a necessary channel condition for the application of SIC between $d_{2}$ and $b$ is identified from (13):

$$
h_{b, d_{1}} h_{d_{2}, u}>h_{d} h_{b, u}
$$

Note that if (23) is false, (13) becomes impossible to satisfy no matter $P_{1}$ and $P_{2}$; however, when (23) is true, (13) can be held true under an adequate power play between $P_{1}$ and $P_{2}$.

We now move to the PMC and SIC conditions for the decoding of $s_{2}$ and $s_{u}$ at the level of $b$ and $d_{1}$ respectively, i.e. (8), (10), (7) and (9). By adding (8) multiplied by $h_{d}$ to (10) multiplied by $h_{b, d_{2}}, P_{2}$ is eliminated to yield:

$$
P_{u}\left(h_{d_{1}, u} h_{b, d_{2}}-h_{b, u} h_{d}\right)>P_{1}\left(h_{b, d_{1}} h_{d}+\eta_{1} h_{b, d_{2}}\right),
$$

which can be further transformed into:

$$
\begin{aligned}
& P_{1}\left(\eta_{1} h_{b, d_{2}}-h_{b, d_{1}} h_{d}\right)+P_{u}\left(h_{d_{1}, u} h_{b, d_{2}}-h_{b, u} h_{d}\right)>2 P_{1} \eta_{1} h_{b, d_{2}} \\
& \quad \Rightarrow P_{1}\left(\eta_{1} h_{b, d_{2}}-h_{b, d_{1}} h_{d}\right)+P_{u}\left(h_{d_{1}, u} h_{b, d_{2}}-h_{b, u} h_{d}\right)>0 .
\end{aligned}
$$

Thus, the PMCs (8) and (10) imply (7). In fact, not only do they imply the rate condition, but it is clear that the PMCs represent more restrictive constraints than rate conditions. Finally, eliminating $P_{u}$ from the PMCs through the combination of (8) multiplied by $h_{d_{1}, u}$ with $(10)$ multiplied by $h_{b, u}$ yields:

$$
P_{2}\left(h_{b, d_{2}} h_{d_{1}, u}-h_{d} h_{b, u}\right)>P_{1}\left(h_{b, d_{1}} h_{d_{1}, u}+\eta_{1} h_{b, u}\right),
$$

which can be rearranged into:

$$
\begin{gathered}
P_{2}\left(h_{d_{1}, u} h_{b, d_{2}}-h_{b, u} h_{d}\right)+P_{1}\left(h_{d_{1}, u} h_{b, d_{1}}-h_{b, u} \eta_{1}\right)>2 P_{1} h_{b, d_{1}} h_{d_{1}, u} \\
\Rightarrow 9 \text {. }
\end{gathered}
$$

Once again, the PMCs for the decoding of $s_{2}$ and $s_{u}$ at $b$ and $d_{1}$ imply their rate condition counterparts. Note that a new necessary channel condition appears from eqs. 24) and 25):

$$
h_{d_{1}, u} h_{b, d_{2}}>h_{b, u} h_{d}
$$

Also, the combinations of (12) with (10), and (14) with (8), while eliminating $P_{u}$, give the following conditions:

$$
\begin{gathered}
P_{1}\left(h_{b, d_{1}} h_{d_{1}, u}-\eta_{1} h_{b, u}\right)>P_{2} h_{d} h_{b, u} \\
P_{2}\left(h_{b, d_{2}} h_{d_{2}, u}-\eta_{2} h_{b, u}\right)>P_{1}\left(h_{b, d_{1}} h_{d_{2}, u}+h_{d} h_{b, u}\right)
\end{gathered}
$$

These inequalities yield two other necessary, but not sufficient, channel conditions for the application of full SIC to the system:

$$
\begin{aligned}
& h_{b, d_{1}} h_{d_{1}, u}>\eta_{1} h_{b, u} \\
& h_{b, d_{2}} h_{d_{2}, u}>\eta_{2} h_{b, u}
\end{aligned}
$$

Repeating the same procedure for the second decoding order delivers the same results: 1) the PMCs encompass the rate conditions, 2) the same necessary four channel conditions are obtained. Therefore, in FD-SIC, the system checks the validity of eqs. (23) and 26) to (28) prior to solving the PA problem for each decoding order. The problem in (1) is only equipped with the PMC set corresponding to the decoding order (i.e. eqs. (8), (10), (12) and (14), or eqs. (19) to (22), in addition to constraints eqs. (1a) to $1 \mathrm{~d}$. Because of the important number 
of constraints, the problem is directly fed to a numerical solver for resolution. If the channel conditions do not comply or no solution is obtained for (1), the FD-SIC algorithm reverts to the FD-NoSIC procedure described in Section II-A1, In the next section, the problem solution for the case of HD-SIC is developed.

\section{HD-SIC SCENARIO}

Consider the first half time slot, where $u$ and $d_{1}$ are transmitting and $b$ and $d_{2}$ are receiving. The rate conditions for the decoding of $s_{1}$ at the level of $b$, and $s_{u}$ at the level $d_{2}$, are derived from $\operatorname{SINR}_{b}^{s_{1}}>\operatorname{SINR}_{d_{2}}^{s_{1}}$ and $\operatorname{SINR}_{d_{2}}^{s_{u}}>\operatorname{SINR}_{b}^{s_{u}}$ respectively. This situation is equivalent to the case of two different radio resource heads (RRHs) transmitting the two signals to two separate receivers, and was studied in [17]. It was shown that the SINR conditions simply lead to the necessary condition (23) obtained in section IV] of the present paper: $h_{b, d_{1}} h_{d_{2}, u}>h_{d} h_{b, u}$. The PMCs for $s_{u}$ and $s_{1}$ at the level of $d_{2}$ and $b$ are given by:

$$
\left.\begin{array}{l}
P_{u, 1} h_{d_{2}, u}>P_{1} h_{d} \\
P_{1} h_{b, d_{1}}>P_{u, 1} h_{b, u}
\end{array}\right\} \Rightarrow A=\frac{h_{d}}{h_{d_{2}, u}}<\frac{P_{u, 1}}{P_{1}}<\frac{h_{b, d_{1}}}{h_{b, u}}=B
$$

We note that $(23)$ is contained in $(29)$, since $(23)$ is equivalent to $A<B$. Therefore, just like for FD-SIC, the PMCs encompass the rate conditions while being more restrictive. Problem (1) now only includes the additional constraint (29) for the first time slot. The HD-SIC rate expressions are as follows:

$R_{D 2 D, 1}=R_{d_{2}}=B \log _{2}\left(1+\frac{P_{1} h_{d}}{\sigma^{2}}\right), R_{u, 1}=B \log _{2}\left(1+\frac{P_{u, 1} h_{b, u}}{\sigma^{2}}\right)$

Maximizing $R_{d_{2}}$ lies in the increase of $P_{1}$, and reaching $R_{u, \text { min }}$ is achieved by setting $P_{u, 1}$ to $P_{u, \text { min }}=\left(2^{\frac{R_{u, \text { min }}}{B}}-\right.$ 1) $\sigma^{2} / h_{b, u}$. However, due to the PMCs, the increase in $P_{1}$ is very likely to increase $P_{u, 1}$ according to the range of allowed values in (29), leading to an excess of CU rate. Since maximization of network throughput (i.e. sum of D2D and $\mathrm{CU}$ rates) is not the objective of this study, we select from the range of admissible $R_{u, 1}$ values, the one closest to $R_{u, \min }$. With that criterion in mind, the D2D rate maximization power allocation problem is solved by increasing $P_{1}$ as much as possible (possibly until $P_{1, \text { max }}$ ) and adjusting $P_{u, 1}$ accordingly. The PA procedure, illustrated in Fig. 2, is as follows: if $P_{1, \text { max }}<P_{u, \text { min }} / A$, keep the couple $\left(P_{1}=P_{1, \text { max }}, P_{u, 1}=\right.$ $\left.P_{u, \text { min }}\right)$. This case is represented by the example $P_{1, \text { max }}^{1}$ on the horizontal blue line in Fig. 2. If this is not the case, check if $A P_{1, \text { max }}>P_{u, \max }$. If yes (cf. example $P_{1, \text { max }}^{3}$ in Fig. 2 , the solution is $\left(P_{u, \max } / A, P_{u, \max }\right)$; if not (cf. example $\left.P_{1, \text { max }}^{2}\right)$, the solution is $\left(P_{1, \max }, A P_{1, \max }\right)$. Restricting the solution space to the blue lines in Fig. 2 guarantees that the $\mathrm{CU}$ always transmits at the minimum necessary power that respects the problem constraints. Note that if $P_{1, \max }$ is too low $\left(<P_{u, \min } / B\right)$, the problem is not feasible even when (23) is verified. For the second time slot, the same methodology is followed, where eq. 26 reappears as a necessary channel condition. In this case, the PMCs are:

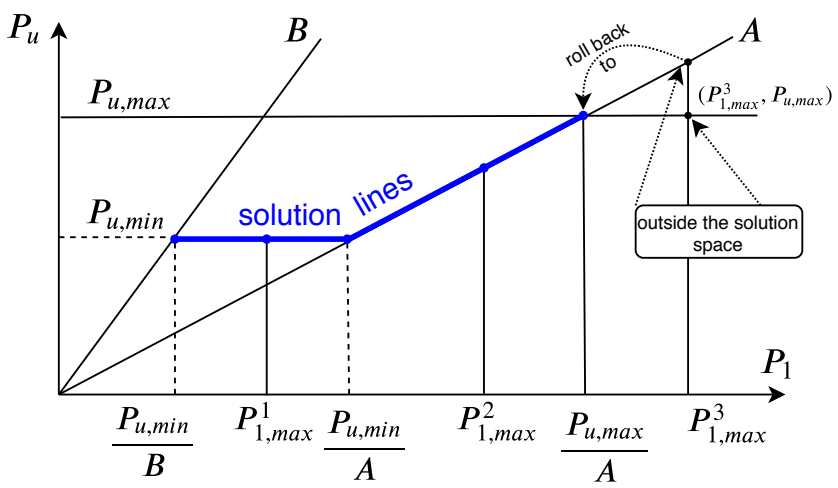

Figure 2: Schematic of the solution space to the HD-SIC PA problem, for different $P_{1, \max }$ values.

$$
A^{\prime}=\frac{h_{d}}{h_{d_{1}, u}}<\frac{P_{u, 2}}{P_{2}}<\frac{h_{b, d_{2}}}{h_{b, u}}=B^{\prime}
$$

As a conclusion, in the HD-SIC scenario, the system checks for the validity of the channel condition corresponding to the half time slot before going through the procedure described above. If the channel condition is not favorable or if no solution exists (i.e. $P_{u, \min }>P_{u, \max }$ or $P_{1, \max }<P_{u, \min } / B$ for the first half, and $P_{2, \max }<P_{u, \min } / B^{\prime}$ for the second half), the system reverts to the HD-NoSIC solution of section II-A2 This leads to four combinations of SIC/NoSIC procedures, two for every half time slot, and they are all included in the HD-SIC algorithm.

\section{NUMERICAL RESULTS}

In our simulation setup, the $\mathrm{BS}$ is at the center of a hexagonal cell with an outermost radius of $300 \mathrm{~m}$. The D2D users are randomly located inside a disk of radius $50 \mathrm{~m}$, centered $100 \mathrm{~m}$ apart from the cell center, whereas the $\mathrm{CU}$ is randomly located anywhere within the cell. The propagation model includes large scale fading with a path loss exponent $\alpha=3.76$, and an $8 \mathrm{~dB}$ zero mean lognormal shadowing. The maximum transmit power of the devices and $\mathrm{CU}$ is $24 \mathrm{dbm}$. The UL subband bandwidth is $156.25 \mathrm{kHz}$, with a noise power of $-122 \mathrm{dbm}$. The SI cancellation factor $\eta=\eta_{1}=\eta_{2}$ varies between -110 and $-60 \mathrm{~dB}$. The results are averaged over 10000 different realizations of the devices and CU positions. The FD-SIC solutions are obtained using function fmincon from Matlab optimization toolbox.

Hereinafter, "Global" figures present the results averaged over all the simulated D2D-CU triplets, including both SIC success and failure cases (in case of failure, SIC algorithms revert to their NoSIC counterparts). On the other hand, the "SIC-only" figures present the results averaged over the cases of FD-SIC success.

The evolution of D2D rates with $\eta$ is shown in Fig. 3a and $3 \mathrm{~b}$, for a minimum target $\mathrm{CU}$ rate $R_{u, \min }=3$ and 7 


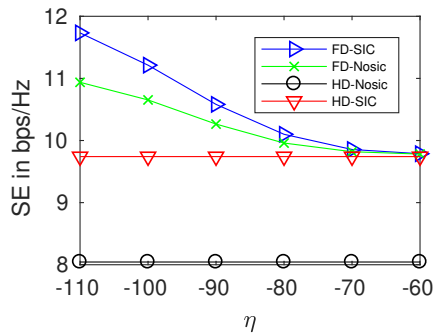

(a) $R_{u, \min }=3 \mathrm{bps} / \mathrm{Hz}$

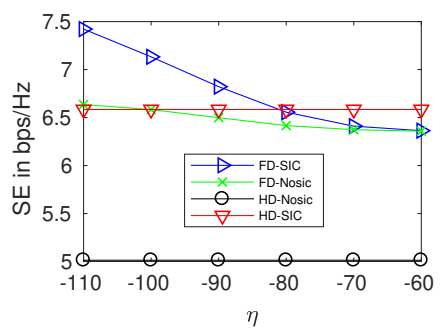

(b) $R_{u, \min }=7 \mathrm{bps} / \mathrm{Hz}$

Figure 3: Global D2D rates as a function of $\eta$.

bps $/ \mathrm{Hz}$ respectively. As expected, the improvement of the SI cancellation capabilities increases the performance of FD algorithms, e.g. the D2D rate of FD-NoSIC in Fig. 3a falls from 10.9 to $9.8 \mathrm{bps} / \mathrm{Hz}$ when $\eta$ varies between -110 and $-60 \mathrm{~dB}$. Also the increase of QoS requirement impacts FD and $\mathrm{HD}$ algorithms by limiting the achieved rate (in FD-NoSIC, for $\eta=-60 \mathrm{~dB}, R_{D 2 D}=6.4 \mathrm{bps} / \mathrm{Hz}$ ), and also by reducing the range of variation of FD algorithms with $\eta$ (for FD-NoSIC, $\Delta R_{D 2 D}=R_{D 2 D}(-110 d B)-R_{D 2 D}(-60 d B)=0.3$ bps for $R_{u, \min }=7 \mathrm{bps} / \mathrm{Hz}$, compared to $1.15 \mathrm{bps} / \mathrm{Hz}$ for $R_{u, \min }=$ $3 \mathrm{bps} / \mathrm{Hz}$ ). On the other hand, the HD curves are independent of $\eta$ since they do not suffer from SI. Note that in the case of NoSIC, FD always outperforms HD since, by shutting down the power of the adequate device, it can revert to the half time slot in HD delivering the best throughput and then extend it to the other half.

Regarding SIC algorithms, it is clear that FD-SIC and HDSIC outperform their NoSIC counterparts. In other terms, the SINR advantages of the SIC operation outweigh the burden incurred by the additional PMC constraints on the solution of problem (11). The gains of FD-SIC with respect to FDNoSIC become marginal when $\eta$ increases, which is due to two phenomenons. First, the number of simulation cases where FD-SIC succeeds (the result of these simulations is isolated in Fig. $4 \mathrm{a}$ and $4 \mathrm{~b}$ ) depends on the value of $\eta$; when $\eta$ increases, harder channel conditions are imposed in eqs. (27) and 28, thus less simulations yield a full SIC (420 cases out of 10000 for $\eta=-110 \mathrm{~dB}$, compared to 32 cases for $\eta=-60 \mathrm{~dB}$ ). This is all the more significant as FD-NoSIC performs rather poorly in these conditions. Second, even when observing the SIConly results in Figs. $4 \mathrm{a}$ and $4 \mathrm{~b}$ a high dependence upon the SI cancellation factor can be seen for FD-SIC with approximately $17 \mathrm{bps} / \mathrm{Hz}$ of dynamic range between $\eta=-60$ and $-110 \mathrm{~dB}$. Since the interference of D2D users is RSI-limited in FD, with the SI cancellation getting better, the D2D rate increases until eventually the RSI's order of magnitude becomes similar to background noise, closing out the gap between HD-SIC (which does not suffer from SI) and FD-SIC between $\eta=-110 \mathrm{~dB}$ and $\eta=-100 \mathrm{~dB}$.

In the "Global" results, the intersection between FD-SIC and HD-SIC only occurs for high values of $R_{u, \min }$ (as in Fig. 3b), taking place around $\eta=-80 \mathrm{~dB}$. The reason behind this is that in Fig. 3a, the original smallest gap between

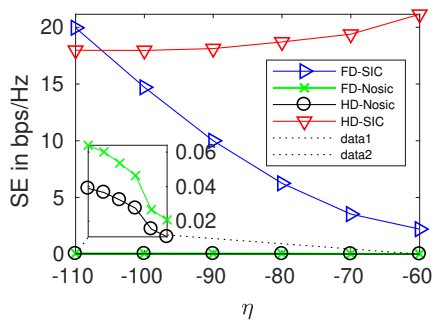

(a) $R_{u, \min }=3 \mathrm{bps} / \mathrm{Hz}$

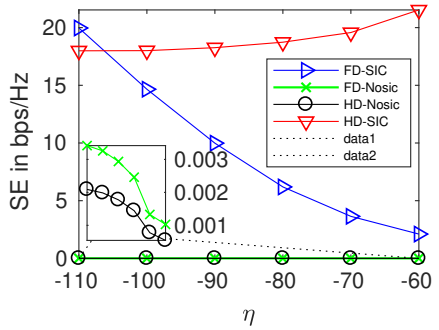

(b) $R_{u, \min }=7 \mathrm{bps} / \mathrm{Hz}$
Figure 4: SIC-only D2D rates as a function of $\eta$.

FD-NoSIC and HD-NoSIC $(\sim 1.9 \mathrm{bps} / \mathrm{Hz})$ is such that the additional rate brought by HD-SIC ( $1.7 \mathrm{bps} / \mathrm{Hz})$ with respect to HD-NoSIC is not sufficient to have an intersection. However, when $R_{u, \min }=7 \mathrm{bps} / \mathrm{Hz}$, the FD/HD-NoSIC gap decreases $(\sim 1.3 \mathrm{bps} / \mathrm{Hz})$ while the additional rate of $\mathrm{HD}$ SIC is almost unchanged $(\sim 1.6 \mathrm{bps} / \mathrm{Hz})$, leading to the intersection mentioned previously. Fig. $3 \mathrm{~b}$ clearly shows that no transmission mode is absolutely better than the other in the "Global" SIC scenario, as opposed to the supremacy of FD-NoSIC with regards to HD-NoSIC.

Moreover, it is observed in Figs. $3 \mathrm{a}$ and $3 \mathrm{~b}$ that the improvement of FD-SIC with respect to FD-NoSIC is virtually the same, independently of the required QoS (around $0.8 \mathrm{bps} / \mathrm{Hz}$ for $\eta=-110 \mathrm{~dB}$ ). This is even clearer in Fig. $4 \mathrm{a}$ and $4 \mathrm{~b}$ which are nearly identical despite the different required rates. To understand this behavior, attention is drawn to Fig. 2 where the optimal HD-SIC PA is depicted: whether $P_{1, \max }$ is such that the solution is in the order of $P_{1, \text { max }}^{1}, P_{1, \text { max }}^{2}$ or $P_{1, \text { max }}^{3}$, increasing $R_{u, \min }$ simply raises the horizontal $P_{u, \min }$ line. Since the optimal PA is obtained from the intersection of the blue segments with the line $P_{1}=\min \left(P_{1, \max }, P_{u, \max } / A\right)$, the abscissa of the optimal PA $\left(P_{1}\right)$ is not affected in any ways by $P_{u, \min }$, therefore the D2D rate is unchanged. This is the same for the case of FD-SIC, leading to the same independence of the D2D rate from $R_{u, \min }$.

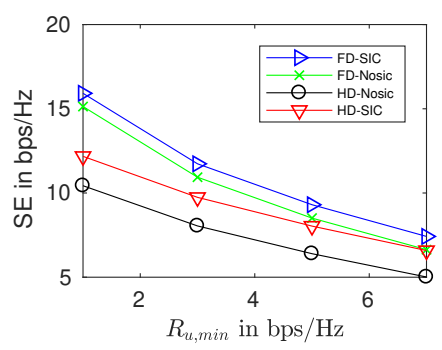

(a) Global

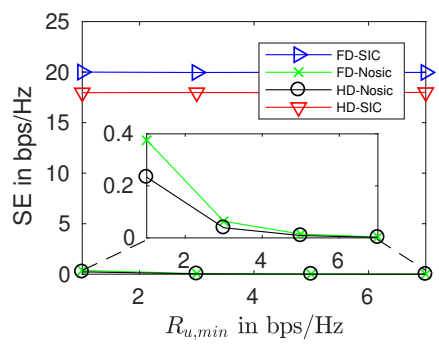

(b) SIC-only
Figure 5: D2D rates as a function of $R_{u, \min }$ for $\eta=-110 \mathrm{~dB}$.

Finally, Fig. 5 shows the evolution of the D2D rates as a function of the $\mathrm{CU}$ target rate, for $\eta=-110 \mathrm{~dB}$. As expected, the global D2D rate of all the techniques decreases with $R_{u, m i n}$, as shown in Fig. 5a, with a clear advantage of FD-SIC over all other methods. The gain of FD-SIC over FD-NoSIC and HD-SIC can reach respectively 0.75 and 3.7 
bps/Hz. Based on the preceding explanations, the D2D rates of SIC-only results are practically independent from $R_{u, \min }$, with $19.9 \mathrm{bps} / \mathrm{Hz}$ gain for FD-SIC over FD-NoSIC and 18 bps/Hz for HD-SIC over HD-NoSIC.

\section{CONCLUSION}

This paper studied the application of NOMA mutual SIC between D2D users and a cellular user in a full-duplex underlay system. The necessary conditions were derived for the SIC feasibility and incorporated in the corresponding power allocation problems. Simulation results confirm the usefulness of mutual SIC in always enhancing performance with respect to the classical NoSIC strategy in both HD and FD. FD-SIC and HD-SIC are shown to deliver the best results depending on the SI cancellation factor: FD-SIC is the best performing algorithm for important SI cancellation factors, while HD-SIC is the best algorithm for low SI cancellation performance. As a future work, adaptive mode selection and channel assignment between FD-SIC and HD-SIC for multiple D2D-CU triplets in a cell will be considered, with the aim of determining efficient geographical and positioning criteria.

\section{ACKNOWLEDGMENT}

This work has been funded with support from IMT Atlantique and the Lebanese University.

\section{REFERENCES}

[1] Cisco, "Cisco Visual Networking Index: Forecast and Trends, 20182023," Mar. 2020.

[2] K. Doppler, M. Rinne, C. Wijting, C. B. Ribeiro, and K. Hugl, "Deviceto-Device Communication as an Underlay to LTE-advanced networks," IEEE Commun. Mag., vol. 47, no. 12, pp. 42-49, Dec 2009.

[3] K. E. Kolodziej, J. G. McMichael, and B. T. Perry, "Multitap RF Canceller for In-Band Full-Duplex Wireless Communications," IEEE Trans. on Wireless Commun., vol. 15, no. 6, pp. 4321-4334, June 2016.

[4] L. Laughlin, C. Zhang, M. A. Beach, K. A. Morris, and J. Haine, "A Widely Tunable Full Duplex Transceiver Combining Electrical Balance Isolation and Active Analog Cancellation," in 2015 IEEE 81st Veh. Technol. Conf. (VTC Spring), May 2015, pp. 1-5.

[5] Y. Saito, A. Benjebbour, Y. Kishiyama, and T. Nakamura, "System-Level Performance Evaluation of Downlink Non-Orthogonal Multiple Access (NOMA)," in 2013 IEEE 24th Annual Int. Symp. on Personal, Indoor, and Mobile Radio Comm. (PIMRC), Sep 2013, pp. 611-615.
[6] J. Farah, E. Sfeir, C. Abdel Nour, and C. Douillard, "New Resource Allocation Techniques for Base Station Power Reduction in Orthogonal and Non-Orthogonal Multiplexing Systems," in 2017 IEEE Int. Conf. on Commun. Workshops (ICC Workshops), May 2017, pp. 618-624.

[7] M. J. Youssef, J. Farah, C. Abdel Nour, and C. Douillard, "Resource Allocation for Mixed Traffic Types in Distributed Antenna Systems Using NOMA," in 2018 IEEE 77th Veh. Technol. Conf. (VTC fall), Aug. 2018, pp. 1-5.

[8] Z. Ding, Z. Yang, P. Fan, and H. V. Poor, "On the Performance of NonOrthogonal Multiple Access in 5G Systems with Randomly Deployed Users," IEEE Signal Process. Lett., vol. 21, no. 12, pp. 1501-1505, Dec. 2014.

[9] Y. Saito, Y. Kishiyama, A. Benjebbour, T. Nakamura, A. Li, and K. Higuchi, "Non-Orthogonal Multiple Access (NOMA) for Cellular Future Radio Access," in 2013 IEEE 77th Veh. Tech. Conf. (VTC Spring), June 2013, pp. 1-5.

[10] Z. Zhang, Z. Ma, M. Xiao, Z. Ding, and P. Fan, "Full-Duplex Deviceto-Device-Aided Cooperative Nonorthogonal Multiple Access," IEEE Trans. on Veh. Technol., vol. 66, no. 5, pp. 4467-4471, May 2017.

[11] W. Sun, E. G. Ström, F. Brännström, Y. Sui, and K. C. Sou, "D2D based V2V Communications with Latency and Reliability Constraints," in 2014 IEEE Globecom Workshops (GC Wkshps), Dec 2014, pp. 14141419.

[12] W. Sun, D. Yuan, E. G. Ström, and F. Brännström, "Cluster-Based Radio Resource Management for D2D-Supported Safety-Critical V2X Communications," IEEE Trans. on Wireless Commun., vol. 15, no. 4, pp. 2756-2769, April 2016.

[13] J. Chen, J. Jia, Y. Liu, X. Wang, and A. H. Aghvami, "Optimal Resource Block Assignment and Power Allocation for D2D-Enabled NOMA Communication," IEEE Access, vol. 7, pp. 90 023-90 035, 2019.

[14] Y. Pan, C. Pan, Z. Yang, and M. Chen, "Resource Allocation for D2D Communications Underlaying a NOMA-Based Cellular Network," IEEE Wireless Commun. Lett., vol. 7, no. 1, pp. 130-133, Feb 2018.

[15] J. Zhao, Y. Liu, K. K. Chai, Y. Chen, and M. Elkashlan, "Joint Subchannel and Power Allocation for NOMA Enhanced D2D Communications," IEEE Trans. on Commun., vol. 65, no. 11, pp. 5081-5094, Nov 2017.

[16] S. Alemaishat, O. A. Saraereh, I. Khan, and B. J. Choi, "An Efficient Resource Allocation Algorithm for D2D Communications Based on NOMA," IEEE Access, vol. 7, pp. 120 238-120 247, 2019.

[17] J. Farah, A. Kilzi, C. Abdel Nour, and C. Douillard, "Power Minimization in Distributed Antenna Systems Using Non-Orthogonal Multiple Access and Mutual Successive Interference Cancellation," IEEE Trans. on Veh. Technol., vol. 67, no. 12, pp. 11873-11885, Dec. 2018.

[18] A. Kilzi, J. Farah, C. A. Nour, and C. Douillard, "New Power Minimization Techniques in Hybrid Distributed Antenna Systems With Orthogonal and Non-Orthogonal Multiple Access," IEEE Trans. on Green Commun. and Netw., vol. 3, no. 3, pp. 679-690, Sep. 2019.

[19] A. Kilzi, J. Farah, C. Abdel Nour, and C. Douillard, "Mutual Successive Interference Cancellation Strategies in NOMA for Enhancing the Spectral Efficiency of CoMP Systems," IEEE Trans. on Commun., vol. 68, no. 2, pp. 1213-1226, Feb 2020.

[20] H. Chour, F. Bader, Y. Nasser, and O. Bazzi, "GALEN: A Geometric Framework for Global Optimal Power Allocation in a Full Duplex D2D Network," in 2019 IEEE Wireless Commun. and Netw. Conf. (WCNC), April 2019, pp. 1-7. 